Abstract for COMPLAS 2021/Composite 2021

\title{
Revised boundary conditions for FE-MD multiscale coupling of amorphous polymers
}

\author{
M. Ries ${ }^{1, a}$, Y. Jain ${ }^{2}$, P. Steinmann ${ }^{1}$, S. Pfaller ${ }^{1}$ \\ ${ }^{1}$ Institute of Applied Mechanics Friedrich-Alexander University Erlangen-Nürnberg, Egerlandstr. 5 \\ 91058 Erlangen \\ amaximilian.ries@fau.de
}

${ }^{2}$ Theoretical Physical Group TU Darmstadt, Alarich-Weiss-Str. 864287 Darmstadt, y.jain@theo.chemie.tu-darmstadt.de

keywords: multiscale simulation, amorphous polymers, FE-MD coupling

Due to their versatility, polymer nanocomposites have become an indispensable class of materials in recent years. Classical simulation approaches like the Finite Element method (FE) often struggle to predict such composite materials' behavior since they cannot account for molecular effects taking place, particularly in the additives' vicinity. To incorporate these effects, many multiscale formulations coupling continuum mechanics with particle descriptions have been proposed. One promising candidate, focusing on amorphous polymers, is the Capriccio Method introduced by Pfaller et al. [1]. They implement a coupling of FE and molecular dynamics (MD) by introducing a handshake region, the so-called bridging domain where information is transferred via virtual particles. The method has proved its capabilities in various studies, e.g., deriving nanocomposites' interphase properties [2,3]. So far, the Capriccio method relies on stochastic boundary conditions (SBC) to confine the particles and create a thermodynamic state. However, these SBCs require cutting any protruding molecule and thus an unphysical change of the microstructure. To address this problem, we extend the SBCs by adding an outer layer of particles governed by the continuum deformation enabling us to generate coupled FE-MD samples whose density matches a periodic MD solution. Combining these new boundary conditions with the inelastic extension of the Capriccio method proposed in [4] allows us to predict amorphous polymers' stress-strain behavior under arbitrary loading accurately. This extension enhances the Capriccio method's performance and will be useful in future studies of polymer nanocomposites.

[1] Pfaller, S.; Rahimi, M.; Possart, G.; Steinmann, P.; Müller-Plathe, F. \& Böhm, M. An Arlequin based method to couple molecular dynamics and finite element simulations of amorphous polymers and nanocomposites Computer Methods in Applied Mechanics and Engineering, 2013 , 260 , 109129.

[2] Pfaller, S.; Possart, G.; Steinmann, P.; Rahimi, M.; Müller-Plathe, F. \& Böhm, M. Investigation of interphase Effects in Silica-Polystyrene Nanocomposites Based on a Hybrid Molecular-DynamicsFinite-Element Simulation Framework Physical Review E, 2016 , 93 , 052505.

[3] Ries, M.; Possart, G.; Steinmann, P.; \& Pfaller, S. A coupled MD-FE methodology to characterize mechanical interphases in polymeric nanocomposites. in preparation.

[4] Zhao, W.; Steinmann, P.; \& Pfaller, S., A particle-continuum coupling method for multiscale simulations of viscoelastic-viscoplastic amorphous glassy polymers. submitted. 




Register for free at https//www.scipedia.com to download the version without the watermark 